\title{
L’hépatite $C$, un enjeu majeur de santé publique
}

\section{Isabelle Fouchard-Hubert}

Praticien hospitalier

Service d'hépato-gastro-entérologie, Centre hospitalier universitaire d'Angers, 4 rue Larrey, 49933 Angers cedex 9, France

Adresse e-mail : ishubert-fouchard@chu-angers.fr (I. Fouchard-Hubert).

\section{Résumé}

L'hépatite Chronique virale $\mathrm{C}$ affecte 71 millions de personnes dans le monde, qui se sont contaminées essentiellement par voie sanguine. Elle est responsable d'une surmortalité liée principalement à la cirrhose, puis à l'hépatocarcinome, ce qui en fait un problème majeur de santé publique. Néanmoins, il s'agit d'une maladie virale dont il est possible de guérir dans plus de $95 \%$ des cas. Ainsi, elle pourrait être éradiquée à l'horizon de quelques années.

(c) 2018

Mots clés - contamination sanguine ; cirrhose ; guérison ; hépatite $C$

Summary à venir

(C) 2018

Keywords à venir

Après la découverte du virus de l'hépatite B (VHB) en 1968, il a paru évident, dans les années 1970, qu'un autre virus était responsable d'hépatite post-transfusionnelle dite "non A non B". Mais ce n'est qu'en 1989 que les anticorps dirigés contre le virus dénommé "hépatite C" (VHC) ont été découverts et qu'un test de dépistage sanguin a été mis au point [1].

\section{T1 Modes de contamination, passés et actuels}

TEG1 Le VHC est transmissible essentiellement par voie sanguine, par contact direct ou indirect avec du sang contaminé. Dans les années 1960-1990, les modes de contamination les plus répandus 
étaient la transfusion de sang et de produits dérivés du sang [2] ainsi que l'usage de drogue par voie intraveineuse via le partage de matériel (seringue, cuillère, filtre, eau, coton) [3]. D'autres modes ont cependant été décrits : sniff (partage de paille), exposition nosocomiale en cas d'usage de matériel mal désinfecté, de malade en malade par l'intermédiaire d'objets souillés ou par le personnel soignant, mais aussi par voie iatrogène (tatouage, piercing...). La transmission du VHC de la mère à l'enfant, si elle est possible, est rare : $5 \%$ au moment de l'accouchement [4]. Enfin, la transmission sexuelle chez les couples hétérosexuels monogames est quasi nulle et il n'y a pas lieu de préconiser I'usage du préservatif [5].

TEG1 En France, grâce aux mesures successives de dépistage des donneurs de sang, le risque actuel de transmission par transfusion est rarissime, estimé à un sur sept millions de transfusions, soit un don infecté tous les trois à quatre ans [6]. En revanche, malgré la politique de réduction des risques entreprise depuis 1987, l'épidémie d'hépatite C persiste chez les usagers de drogue, même si la prévalence dans ce milieu a diminué.

TEG1 Enfin, depuis les années 2000, une épidémie de VHC frappe le milieu homosexuel masculin, en Europe et aux États-Unis, que les patients soient ou non porteurs du virus de l'immunodéficience humaine $(\mathrm{VIH})$. Ce phénomène est lié à des pratiques sexuelles traumatiques, le plus souvent sous l'emprise de produits (chemsex) [7].

\section{T1 Épidémiologie}

TEG1 Alors que l'épidémie, après avoir atteint un pic, recule depuis la deuxième moitié du $\mathrm{xx}^{\mathrm{e}}$ siècle dans le monde, 1,75 million d'individus auraient encore été infectés par le VHC en 2015. Une toxicomanie intraveineuse ou des pratiques d'injection non sécurisées (vaccins par exemple) seraient en cause. Selon le rapport de l'Organisation mondiale de la santé (OMS) de 2017, 71 millions de personnes sont porteuses du VHC, soit $1 \%$ de la population mondiale. De plus, 2,3 millions de patients sur les 36,7 millions porteurs du VIH seraient co-infectés avec le VHC. A priori, $20 \%$ seulement des sujets porteurs du VHC sont diagnostiqués et $7 \%$ ont été traités [8].

TEG1 La France est un pays de faible endémie pour le VHC, dont la prévalence continue à diminuer avec le temps. En 2004, celle du VHC avec acide ribonucléique (ARN) détectable a été évaluée à 0,53 \%, puis, en 2011, à 0,42 \%, soit 190000 personnes [9]. La prévalence du VHC parmi la population toxicomane intraveineuse a été estimée à $60 \%$ et $28 \%$ chez les moins de 30 ans en 2004 [10], puis à $44 \%$ et $19 \%$ chez les moins de 30 ans en 2011 [11]. Toutefois, en 2014, le nombre de personnes porteuses du VHC non diagnostiquées restait élevé : 74 000, contre 100000 en 2004 [12]. Malgré une activité de dépistage importante et en constante augmentation depuis les années 2000, celui-ci reste perfectible. C'est la raison pour laquelle la question d'un dépistage populationnel du VHC en complément du dépistage ciblé a été soulevée, mais n'a toujours pas été validée à ce jour. Aux États-Unis, depuis 2012, le dépistage concerne l'ensemble des personnes nées entre 1945 et 1965. 


\section{T1 Histoire naturelle}

TEG1 L'incubation de l'hépatite $\mathbf{C}$ aiguë varie de quatre à douze semaines. Le plus souvent, elle passe inaperçue. Les symptômes, s'ils existent, ne sont pas spécifiques au VHC - nausées, fatigue, douleur de l'hypochondre droit - et durent en général deux à douze semaines. Ces signes peuvent précéder l'apparition d'urines foncées, puis d'un ictère. L'infection virale C persiste, dans 50 à $75 \%$ des cas, six mois après la contamination, il est alors question d'hépatite $C$ chronique $[13,14]$. Les personnes qui ont éliminé spontanément le VHC présentent néanmoins une sérologie virale $C$ positive, bien que la charge virale ne soit pas détectable. Ils sont considérés comme guéris mais peuvent être recontaminés lors d'un nouveau contact avec le virus. Les facteurs favorisant un passage à la chronicité sont partiellement connus : un âge élevé au moment de la contamination, une immunodépression telle que l'infection par le VIH, l'absence de symptômes au moment de l'hépatite aiguë et le génotype IL 28B c/c en font partie [15].

\section{TEG1 L'infection chronique virale $C$ est responsable d'une activité nécrotico-inflammatoire} hépatique liée à la persistance du virus dans l'organisme et à l'activation du système immunitaire. Il en résulte la production d'une fibrose hépatique. La fibrose n'est pas spécifique du VHC, c'est une réaction inflammatoire en réponse à une agression qui peut être infectieuse (virale) et/ou toxique (alcool) et/ou métabolique (stéatose, surcharge en fer). À terme, elle peut évoluer vers une cirrhose. La vitesse de progression de la fibrose varie beaucoup d'un patient à l'autre et peut s'accélérer avec le temps chez un même individu. Globalement, il est estimé que 10 à $20 \%$ des patients développent une cirrhose sur plusieurs décennies [16]. Les facteurs favorisants sont liés à l'hôte ou à l'environnement, en particulier la consommation excessive d'alcool et le syndrome métabolique. Le fait d'avoir une cirrhose expose le patient à des complications mortelles, insuffisance hépatocellulaire et hépatocarcinome, dont la fréquence est de 2 à $5 \%$ par an [17]. La consommation d'alcool ou de tabac et le syndrome métabolique ont été identifiés comme étant des facteurs de risque pour la survenue d'un hépatocarcinome sur cirrhose préexistante. Il a été estimé qu'en France, 2600 patients étaient décédés en 2001 de cirrhose virale C, un chiffre qui n'a pas été actualisé depuis.

TEG1 L'hépatite Chronique virale $\mathrm{C}$ est, en France, la deuxième cause de cirrhose après la consommation excessive d'alcool. C'est également la deuxième cause de transplantation hépatique. La cirrhose est potentiellement réversible grâce au traitement du VHC. Classiquement, I'hépatite Chronique virale $C$ est peu symptomatique, même au stade de cirrhose, ce qui explique qu'un grand nombre de personnes ne soient pas encore dépistées. Du point de vue biologique, il est en général observé une augmentation modérée des transaminases de deux à trois fois la normale. Toutefois, elles peuvent être strictement normales.

TEG1 Le VHC peut être également responsable de manifestations extra-hépatiques auto-immunes ou inflammatoires ou encore métaboliques : atteintes cardiovasculaires, insuffisance rénale, troubles anxiodépressifs, fatigue altérant la qualité de vie, cryoglobulinémie, voire lymphome $B$, diabète... Elles sont indépendantes de la sévérité de la maladie hépatique.

TEG1 Les personnes atteintes du VHC ont ainsi une surmortalité hépatique mais également extrahépatique [18]. 


\section{T1 Diagnostic}

TEG1 Le diagnostic d'hépatite aiguë virale $\mathbf{C}$ se fonde sur la recherche du génome viral $\mathbf{C}$, qui est détectable une à trois semaines avant l'apparition des anticorps dirigés contre le VHC, et donc de la positivité de la sérologie virale $C$. Classiquement, une augmentation importante des transaminases est retrouvée, ce qui est spécifique à une hépatite aiguë.

TEG1 Le diagnostic d'hépatite virale $\mathbf{C}$ chronique est réalisé par une sérologie qui peut être effectuée lors d'un bilan sanguin usuel ou par un test rapide d'orientation diagnostique (Trod) (figure 1). La Haute Autorité de santé (HAS) a recommandé en 2011 la réalisation, après la première sérologie positive, d'une deuxième sérologie virale $C$ lors d'un deuxième prélèvement sanguin [19]. Il est primordial, lors de ce deuxième prélèvement, de réaliser une recherche du génome du virus $C$ par PCR (réaction en chaîne par polymérase) quantitative afin de départager les patients spontanément guéris (charge virale $\mathrm{C}$ indétectable) des patients porteurs du VHC (charge virale $\mathrm{C}$ détectable). Il convient également de vérifier le statut concernant l'hépatite virale $B$ et le virus du sida car des coinfections peuvent exister.

\section{T1 Prise en charge}

TEG1 Une évaluation de la fibrose hépatique est nécessaire chez tout patient porteur du VHC, à la recherche d'éventuelles manifestations hépatiques et notamment d'une cirrhose. Cette évaluation est réalisée par des techniques dites "non invasives" qui ont l'avantage, par rapport à la biopsie hépatique, d'être sans aucun risque. En 2008, la HAS a recommandé de recourir soit à l'un des trois scores sanguins de fibrose (Fibrotest ${ }^{\circ}$, Fibromètre ${ }^{\circ}$, Hépascore ${ }^{\circ}$ ), soit à une mesure de l'élasticité hépatique par une méthode physique (Fibroscan ${ }^{\circ}$ ). Ces tests sont pris en charge à $100 \%$ par la Sécurité sociale. Le diagnostic de maladie hépatique sévère est écarté si les valeurs sont inférieures aux seuils suivants : Fibroscan ${ }^{\circ}<10 \mathrm{kPa}$; Fibrotest ${ }^{\bullet} \leq 0,58$; Fibromètre ${ }^{\circ} \leq 0,786$ [20].

TEG1 En cas de cirrhose, il est nécessaire de programmer une fibroscopie œsogastrique, pour rechercher des varices œsophagiennes ou gastriques liées à l'hypertension portale, ainsi qu'un dépistage d'un hépatocarcinome (incidence annuelle de 3 à $5 \%$ ) par échographie hépatique et bilan sanguin tous les six mois. La surveillance d'un patient cirrhotique doit être maintenue, même après la guérison du VHC, car il persiste un risque d'hépatocarcinome même si celui-ci est diminué. Il convient également de prendre en charge les comorbidités telles que la consommation excessive d'alcool et le surpoids [21].

TEG1 Depuis mars 2017, l'ensemble des patients porteurs du VHC peut accéder à de nouvelles molécules et ce, quel que soit le niveau de fibrose hépatique. Dans certaines situations cliniques, il est nécessaire de valider l'autorisation du traitement en présentant le dossier du patient en réunion de concertation pluridisciplinaire, réunions organisées en général par les centres experts hépatites.

\section{Points à retenir}

- Le virus de l'hépatite $\mathrm{C}(\mathrm{VHC})$ est fréquent et ubiquitaire. 
- L’hépatite C est une maladie virale silencieuse.

- L'intérêt d'un dépistage du VHC au moins une fois dans sa vie est avéré.

- L’hépatite $C$ peut évoluer vers une cirrhose et entraîner des manifestations extra-hépatiques.

- La guérison virale est envisageable grâce à un traitement médical révolutionnaire.

\section{T1 Conclusion}

L'hépatite Chronique virale C est une affection fréquente, peu symptomatique, à l'origine d'un grand nombre de maladies chroniques du foie et de décès (350 000 par an au niveau mondial), mais aussi de complications extra-hépatiques polymorphes.

Cette maladie virale est quasi unique car elle est curable grâce à un traitement médicamenteux. Depuis sa découverte il y a presque 30 ans, des progrès considérables, qui peuvent être qualifiés de révolutionnaires, ont été réalisés. Ils permettent d'envisager une éradication de la maladie à l'échelle planétaire en 2030 , ce qui implique toutefois de mettre en œuvre une politique active de dépistage.

\section{Références}

[1] Choo $Q$, Kuo $G$, Weiner $A$ et al. Isolation of a cDNA clone derived from a blood-borne non-A, nonB viral hepatitis genome. Science. 1989;244(4902):359-62.

[2] Nelson KE, Donahue JG, Stambolis V. Post transfusion hepatitis C virus infection. N Engl J Med. 1992;328:1601-2.

[3] Conry-Cantilena C, Van Raden M, Gibble J et al. Route of infection, viremia, and liver disease in blood donors found to have hepatitis C virus infection. N Engl J Med. 1996;334(26):1961-6.

[4] Zanetti AR, Tanzi E, Newell ML. Mother-to-infant transmission of hepatitis C virus. J Hepatol. 1999;31(Suppl 1):96-100.

[5] Vandelli C, Renzo F, Romanò L et al. Lack of evidence of sexual transmission of hepatitis $C$ among monogamous couples: results of a 10-year prospective follow up study. Am J Gastroenterol. 2004;99(5):855-9.

[6] Pillonel J, Legrand D, Sommen $C$ et al. Surveillance épidémiologique des donneurs de sang et risqué résiduel de transmission du VIH, de l'HTLV, du VHC et du VHB par transfusion sanguine en France entre 2008 et 2010. Bull Epidemiol Hebd. 2012;39-40.

[7] Hagan $H$, Jordan AE, Neurer J et al. Incidence of sexually transmitted hepatitis $C$ virus infection in HIV-positive men who have sex with men. AIDS. 2015;29(17):2335-45. 
[8] Organisation mondiale de la santé (OMS). Global hepatitis report, 2017. Genève: WHO; 2017. www.who-int/hepatitis/publication/global-hepatitis-report2017

[9]. Pioche C, Pelat C, Larsen C et al. Estimation de la prévalence de l'hépatite $C$ en population générale, France métroplitaine, 2011. Bull Epidemiol Hebd. 2016;13-14.

[10] Jauffrey-Roustide $M$, Couturier E, Le Strat $Y$ et al. Estimation de la séroprévalence du VIH et du VHC et profils des usagers de drogue en France, étude INVS-ANRS Coquelicot, 2004. Bull Epidemiol Hebd. 2006;33.

[11] Jauffret-Roustide M, Pillonel J, Weill-Barillet L et al. Estimation de la séroprévalence du VIH et de I'hépatite $C$ chez les usagers de drogues en France - Premiers résultats de l'enquête ANRS-Coquelicot 2011. Bull Epidemiol Hebd. 2013;39-40.

[12] Brouard C, Le Strat Y, Larsen C et al. Estimation du nombre de personnes non diagnostiquées pour une hépatite $\mathrm{C}$ chronique en France en 2014. Implications pour des recommandations de dépistage élargi. Bull Epidemiol Hebd. 2015;19-20.

[13] Seef LB, Hollinger FB, Alter HJ et al. Long term mortality and morbidity of transfusion-associated non-A, non-B and type $C$ hepatitis: a national Heart, Lung, and Blood Institute collaborative study. Hepatology. 2001;33(2):455-63.

[14] Kenny-Walsh E. Clinical outcomes after hepatitis $C$ infection from contamined anti-D immune globulin. Irish Hepatology Research Group. N Engl J Med. 1999;340(16):1228-33.

[15] Santantonio T, Wiegand J, Gerlach JT. Acute hepatitis C: current status and remaining challenges. J Hepatol. 2008;49(4):625-33.

[16] Afdhal NH. The natural history of hepatitis C. Semin Liver Dis. 2004;24(Suppl 2):3-8.

[17] Trinchet JC. Histoire naturelle de l'infection par le virus de I'hépatite C. Gastroenterol Clin Biol. 2002;26(HS 2):144-53.

[18] Cacoub P, Desbois AC, Commarmond C et al. Les atteintes extrahépatiques du virus de I'hépatite C : un concept revisité. Rev Med Int. 2016;37(6):383-6.

[19] Haute Autorité de santé (HAS). Stratégies de dépistage biologique des hépatites virales B et C. Mars 2011. www.has-sante.fr/portail/upload/docs/application/pdf/2011-

05/strategies_de_depistage_biologique_des_hepatites_virales_b_et_c_-_argumentaire.pdf

[20] Association française pour l'étude du foie (AFEF). Recommandations AFEF pour l'élimination de l'infection par le virus de l'hépatite $C$, en France. Mars 2018. https://afef.asso.fr/wpcontent/uploads/2018/06/VF-INTERACTIF-RECO-VHC-AFEF-v2103.pdf

[21] European Association for the Study of the Liver. EASL Recommendations on Treatment of Hepatitis C 2018. J Hepatol. 2018;in Press. https://doi.org/10.1016/j. jhep.2018.03.026 
Déclaration de liens d'intérêts

L'auteur déclare travailler en collaboration avec les laboratoires Gilead, MSD et Abbvie.

\section{Figure}

\section{Fou_fig1}

Figure 1. Test de dépistage rapide de l'hépatite $C$.

(c) Africa Studio/stock.adobe.com

Sur 1 colonne près de son appel

\section{Illustration}

\section{Fou_illus1}

Identifié en 1989, le virus de l'hépatite C est transmissible par contact direct ou indirect avec du sang contaminé.

(c) fotoliaxrender/stock.adobe.com

En début d'article sur 1 colonne + marge en haut (ou une colonne) 


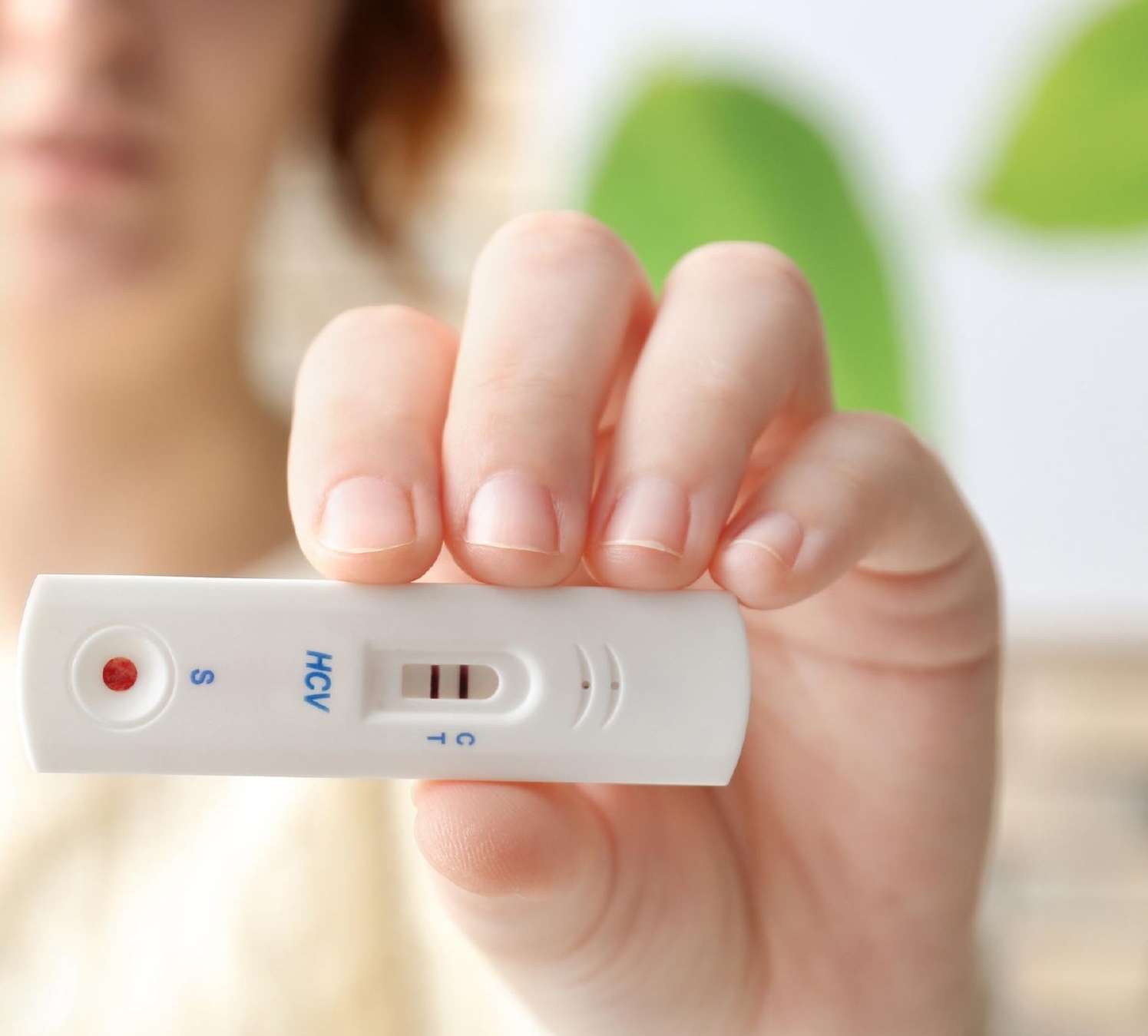


